\title{
Photoperiodic time measurement is maintained in undernourished lambs with delayed puberty*
}

\author{
D. L. Foster and S. M. Yellon† \\ Reproductive Endocrinology Program, Department of Obstetrics and Gynecology, and Division of \\ Biological Sciences, The University of Michigan, Ann Arbor, MI 48109, U.S.A.
}

\begin{abstract}
Summary. Beginning at 42 weeks of age undernourished females that had been maintained outdoors were exposed to long days (15L:9D) or short days (9L:15D). After 6 weeks, both groups were placed on short days, and ad-libitum feeding was begun. Rapid 'catch-up' growth occurred similarly in both groups. However, the response to oestradiol negative feedback regulation of LH secretion differed greatly. Short-day lambs remained hyperresponsive to oestradiol inhibition, and circulating $\mathrm{LH}$ remained low, a condition that typifies immaturity of the system governing LH secretion. In the females exposed to the long-day-short-day sequence, circulating LH began to increase 10 weeks after the end of long days; this change is characteristic of the neuroendocrine alteration that occurs during puberty. These findings indicate that the growth-retarded lamb can differentiate long days from short days, and can therefore continue to accumulate photoperiod information during prolonged periods of undernutrition.
\end{abstract}

\section{Introduction}

Both photoperiod and growth serve as important determinants timing puberty in the female lamb (for review see Foster, Yellon \& Olster, 1985). We have postulated that the long days of spring and summer followed by the shorter days of autumn provide the appropriate daylength cues for first ovulation at 30 weeks of age in well-nourished, spring-born lambs. Experimentally, as little as 1 week of artificial long days will initiate puberty in females otherwise raised in short days from birth; in the absence of long days, lambs reared in short days remain anovulatory during the first year after birth (Yellon \& Foster, 1985). Similarly, lambs raised in the natural photoperiod, but which are severely undernourished, do not begin reproductive cycles during the first year (Foster \& Olster, 1985). Perhaps undernutrition prevents puberty by abolishing the recognition of the long-dayshort-day photoperiod sequence. The present study examined this possibility. Our approach was to expose undernourished, growth-retarded lambs to an appropriate photoperiod sequence for puberty, and then determine whether neuroendocrine sexual maturity could be expressed after the resumption of growth.

\section{Materials and Methods}

\section{General}

The experiment was conducted from January to June 1983 in 12 ovariectomized Suffolk sheep between 42 and 65 weeks of age under controlled lighting. The artificial long days ( $15 \mathrm{~h}$ light:9 h

\footnotetext{
* Reprint requests to: D. L. Foster, Reproductive Endocrinology Program, Room 1101, 300 North Ingalls Building, The University of Michigan, Ann Arbor, MI 48109, U.S.A.

$\dagger$ Present address: Department of Physiology, Division of Perinatal Biology, Loma Linda University School of Medicine, Loma Linda, CA 92350, U.S.A.
} 
dark, lights on 05:00 h EST; 15L:9D) and short days (9L:1 5D, lights on 06:00 h EST) approximated the daylengths that occur during the local summer and winter solstices, respectively (Ann Arbor, MI, $42^{\circ} 18^{\prime} \mathrm{N}$ ). A $7-\mathrm{W}$ red light ( $<0.2 \mathrm{lux}$ ) remained on continuously to provide minimal illumination when entry to the room was necessary during the dark phase of the photoperiod. The females were fed a diet of grain (oats and maize), a commercial ration (18 Lamb, Kent Feeds, Inc., Muscatine, Iowa), and hay. The major components of the commercial ration were as follows: $18 \%$ crude protein, $2 \%$ crude fat, $16 \%$ crude fibre, vitamin $\mathrm{A}$, vitamin $\mathrm{D}_{3}$ and vitamin $\mathrm{E}$. The ratio of oats:maize:commercial ration was 1:1:3.5; water was always provided ad libitum.

Before the experiment, the females had been used to determine the effects of undernutrition on tonic and surge modes of $\mathrm{LH}$ secretion (Foster \& Olster, 1985). In that study, conducted in natural photoperiod, body weight had been maintained at $20 \mathrm{~kg}$ from 10 weeks of age by food restriction, ovaries had been removed at 20 weeks, and Silastic capsules containing oestradiol had been implanted s.c. to induce LH surges at 40 weeks; the capsules were removed 1 week later.

\section{Treatments and hormone assays}

The design of the experiment permitted comparison of the control of $\mathrm{LH}$ secretion during ad-libitum feeding of young females previously exposed to different photoperiods during severe undernutrition. One photoperiod treatment was a long-day-short-day sequence which is known to initiate reproductive cycles in normally growing lambs; the other was continuous short days, a photoperiod that delays puberty (Yellon \& Foster, 1985). A decrease in response to oestradiol inhibition of LH secretion was used as the index for the pubertal transition (Foster \& Ryan, 1979). The details of the experiment, including the time course of the photoperiod treatment and growth curves, are presented below and in Text-fig. 1.

At 42 weeks of age, the 12 food-restricted, ovariectomized females (mean body weight $\sim 21 \mathrm{~kg}$ ) were transferred from natural to artificial photoperiods (Day 0 of experiment). Six of the lambs were placed on long days (Text-fig. 1c); 6 weeks later, the photoperiod was changed to short days for 17 weeks. At the shift from long days to short days, ad-libitum feeding was begun (Text-fig. la). Five days before this light shift, an oestradiol-containing Silastic capsule, which was designed to achieve a circulating oestradiol concentration of $2-4 \mathrm{pg} / \mathrm{ml}$ (Foster, 1984), was inserted s.c. To maintain oestradiol concentrations within these approximate limits during the rapid growth phase of the experiment, an additional oestradiol capsule was implanted when the average body weight exceeded $30 \mathrm{~kg}$ (Text-fig. 1a). The remaining 6 undernourished, ovariectomized females were transferred to artificial short days at 42 weeks of age where they remained for the entire 23 weeks of the experiment (Text-fig. lb). They were placed on the ad-libitum diet and were treated with oestradiol at the same times as those experiencing the long-day-short-day photoperiod sequence. Body weights were measured weekly.

Blood samples $(5 \mathrm{ml})$ were collected by jugular venepuncture twice each week (Monday and Thursday). LH concentrations were determined in duplicate volumes of $50 \mu \mathrm{l}$ or $200 \mu \mathrm{l}$ serum by a radioimmunoassay (Niswender, Reichert, Midgley \& Nalbandov, 1969) which was modified as described previously (Hauger, Karsch \& Foster, 1977). Intra-assay coefficients of variability (CV) for 2 standard sera each analysed 6 times per assay averaged $3.3 \%$ and $5 \%$ at $43 \%$ and $74 \%$ displacement of radiolabelled $\mathrm{LH}$, respectively. Interassay $\mathrm{CV}$ for these same standard sera were $5.4 \%$ and $12.5 \%$, respectively ( 5 assays). The limit of detection ( $95 \%$ confidence limits of buffer controls) averaged $0.30 \mathrm{ng} / \mathrm{ml}$ for $200 \mu \mathrm{l}$ serum. Results are expressed in terms of NIH-LH-S12.

\section{Results}

At 41 weeks of age, before treatments were initiated, the average ( \pm s.e.m.) weight of the chronically undernourished lambs was $21 \cdot 1 \pm 0 \cdot 7 \mathrm{~kg}(\mathrm{~N}=12)($ Text-fig. 1). This low weight was 


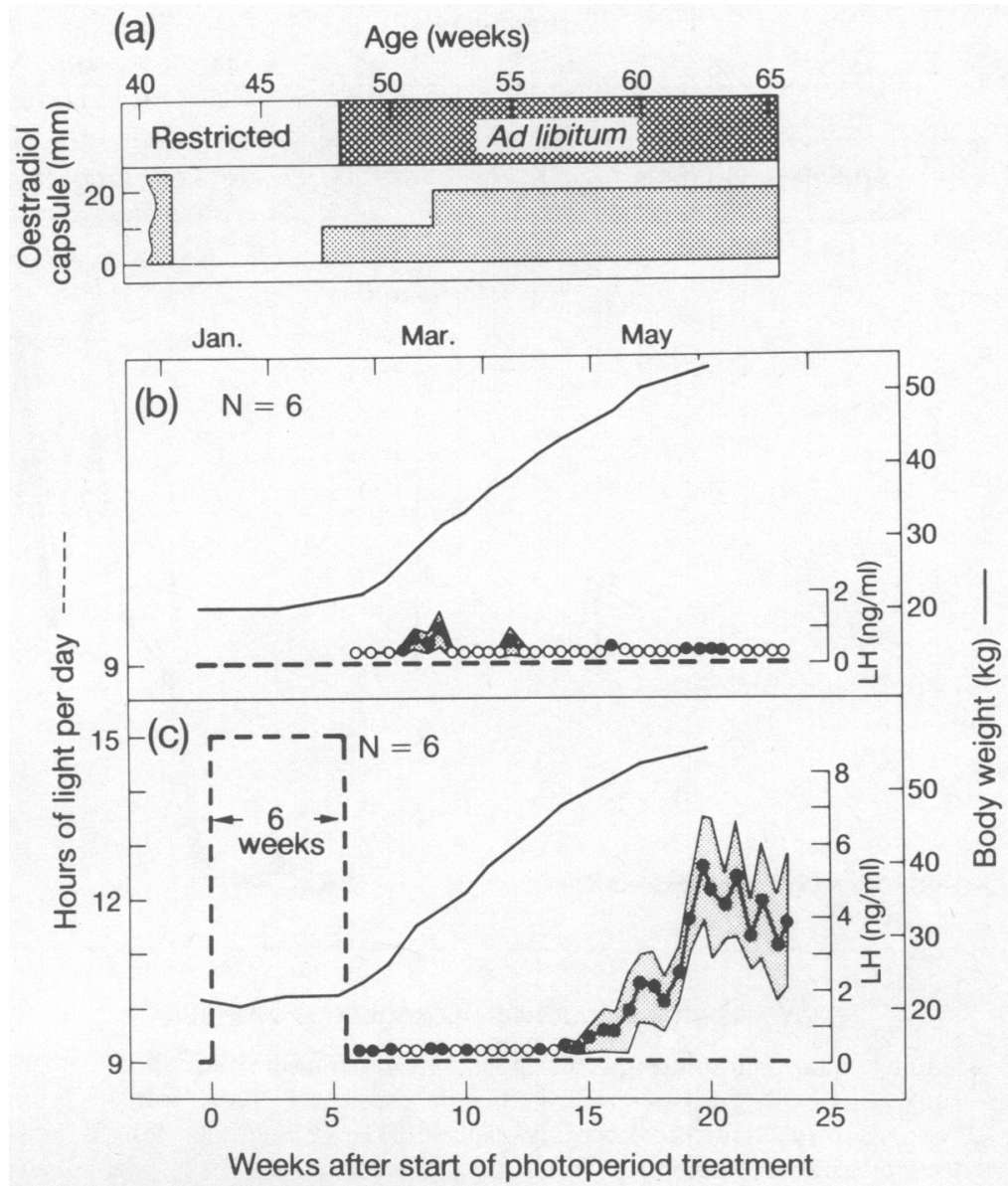

Text-fig. 1. Experimental protocol (a) and results for exposure of undernourished ovariectomized lambs to (b) a short artificial photoperiod or (c) a long photoperiod before ad-libitum feeding in a short photoperiod. Results for serum LH are presented as means ( \pm s.e.m.); open circles designate undetectable $(<0.30 \mathrm{ng} / \mathrm{ml}) \mathrm{LH}$ values.

maintained during continued food restriction for an additional 6 weeks in artificial photoperiods. During subsequent ad-libitum feeding under short days, the females gained an average of $32.5 \mathrm{~kg}$ $(2 \cdot 3 \mathrm{~kg} /$ week, Weeks 7-20).

In ovariectomized females maintained entirely in short days, peripheral concentrations of LH were low or undetectable $(<0.30 \mathrm{ng} / \mathrm{ml})$ in the presence of exogenous oestradiol during ad-libitum feeding (Text-fig. 1b). A different response occurred in the group exposed to long days before ad-libitum feeding under short days (Text-fig. 1c). During the first 10 weeks after completion of the long-day treatment, concentrations of circulating $\mathrm{LH}$ in the presence of exogenous oestradiol were low or undetectable. Thereafter, the response to oestradiol inhibition decreased, and serum $\mathrm{LH}$ values rose several-fold.

The focus of the study was on the LH response to oestradiol inhibition of LH secretion during ad-libitum feeding in short days after exposure to different photoperiods during restricted feeding. However, marked differences were also apparent in the $\mathrm{LH}$ response much earlier. This is shown in Text-fig. 2 during the initial treatments with artificial daylengths when the ovariectomized lambs were still undernourished. Oestradiol capsules were removed after completion of a previous experiment conducted in natural conditions (Foster \& Olster, 1985). Withdrawal of exogenous 


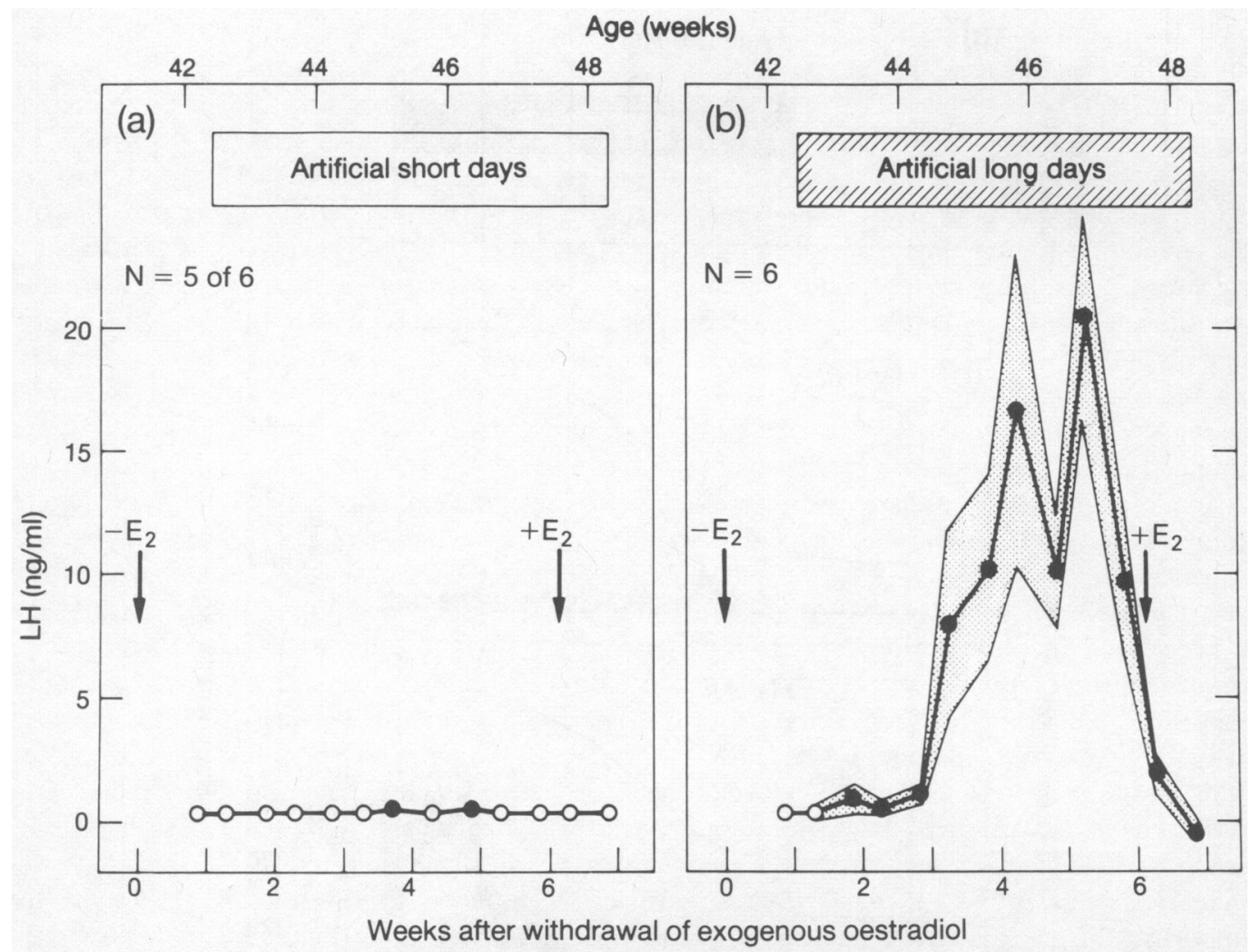

Text-fig. 2. Mean ( \pm s.e.m.) circulating LH concentrations in undernourished, ovariectomized lambs after removal of exogenous oestradiol (Silastic capsule, $E_{2}$ ) and transfer from natural short days (January) to (a) artificial short days (9L:15D) or (b) artificial long days (15L:9D). Open circles designate undetectable $(<0.03 \mathrm{ng} / \mathrm{ml}) \mathrm{LH}$ values.

oestradiol resulted in a pronounced increase in circulating $\mathrm{LH}$ values 2 weeks later during the subsequent long-day treatment (Text-fig. 2b). Reinsertion of the oestradiol implant during long days suppressed circulating LH once again to low levels. In the other experimental group, removal of oestradiol did not result in an increase in mean levels of LH in 5 of the 6 females placed in short days; in the remaining female, an LH rise occurred after withdrawal of the exogenous oestrogen (Text-fig. 2a).

\section{Discussion}

This experiment used the oestradiol-treated, ovariectomized lamb to determine whether severe undernutrition prevents puberty by abolishing the ability to respond to photoperiod. The degree of malnutrition used was sufficient to retard both gain in body weight and increases in skeletal growth (Foster \& Olster, 1985). The results clearly reveal that the growth-retarded lamb can readily detect changes in photoperiod, as determined by changes in circulating LH. First, the response to acute removal of exogenous oestradiol from undernourished lambs was dependent upon daylength; in short days, an LH rise did not occur, whereas oestradiol withdrawal followed by long days produced a several-fold increase in circulating LH. Second, during the period of rapid "catch-up growth', the ability of oestradiol to suppress LH depended upon the photoperiod that was experienced when the females were undernourished. Chronic oestradiol treatment was capable of 
maintaining low concentrations of circulating LH in females housed continuously in short days, but not in those that had been exposed to the 6 weeks of long days before treatment with short days. The 10-12-week interval between the shift from long days to short days and the beginning of the sustained increase in circulating LH in ovariectomized lambs is similar to the latent period for initiation of ovulation in intact lambs after a brief treatment with long days (Yellon \& Foster, 1985). In lambs without ovaries, high responsiveness to exogenous oestradiol, as reflected by low circulating LH, characterizes the anovulatory condition; conversely, increased LH indicates a decreased response to oestradiol inhibition and is considered to reflect the ability of the hypothalamic-pituitary system to support ovarian cyclicity (Foster \& Ryan, 1979). We therefore suggest that if the ovaries had been present instead of the exogenous oestradiol, the females exposed to artificial long days when undernourished would have exhibited ovulatory cycles during their growth spurt in short days; accordingly, those exposed only to short days would have remained anovulatory regardless of level of nutrition.

In a previous study of these ovariectomized lambs under natural photoperiod, the system governing LH secretion was impaired by undernutrition; despite receiving the appropriate photoperiod sequence from the long days of spring and summer and then the short days of autumn and winter, they remained hypersensitive to oestradiol inhibitory feedback (Foster \& Olster, 1985). The ability to keep track of photoperiod during severe malnutrition would offer an explanation for why females of the present study that were treated entirely with short days failed to reduce their response to oestradiol feedback inhibition during the period of 'catch-up' growth (Text-fig. 1b). We propose that exposure of lambs to the natural short days of autumn and winter during the previous study and then to the artificial short days of the present experiment produced photorefractoriness to short days. According to this hypothesis, exposure of the other group of lambs to artificial long days broke the short-day photorefractoriness and allowed the females to respond to short days by decreasing sensitivity to oestradiol negative feedback when they resumed growth (Text-fig. 1c). In support of this explanation are results of other studies of photoperiod requirements for puberty in the well-nourished lamb (Yellon \& Foster, 1985): lambs reared continuously in short days experienced delayed puberty while exposure to a 5-week block of long days, which is of similar duration to that ( 6 weeks) used in the present study, initiated reproductive cycles within the normal age range (25-35 weeks).

Finally, the differential response to acute withdrawal of oestradiol in undernourished, ovariectomized lambs in short days and long days bears upon the above discussion (Text-fig. 2). Perhaps the proposed short-day photorefractoriness accounts for the lack of an increase in circulating $\mathrm{LH}$ in short days after removal of exogenous oestradiol; in such sheep, there seems to be little, if any, ability to secrete $\mathrm{LH}$ in the absence of steroid inhibitory feedback during prolonged exposure to short days. From the response in the other experimental group, it would appear that a relatively brief exposure to long days is necessary to begin to break short-day photorefractoriness in the absence of steroids. After only 2 weeks of long days, the ability to secret LH at high levels was restored. A similar phenomenon has been described in the canary that is refractory to stimulatory photoperiod. Removal of gonadal steroids by castration also does not produce an increase in serum LH; however, a prompt post-castration LH rise will occur if photorefractoriness is broken by exposure of such birds to inhibitory photoperiod (Nicholls \& Storey, 1976). By contrast, the adult ovariectomized ewe, when rendered photorefractory to stimulatory photoperiod by prolonged exposure to short days, can still readily secrete $\mathrm{LH}$ in response to withdrawal of exogenous oestradiol (J. E. Robinson \& F. J. Karsch, unpublished results). It remains to be determined whether nutritional state or maturational state is responsible for the above differences in photoneuroendocrine response between the growth-retarded lamb and the well-fed adult sheep.

The foregoing considerations lead to the conclusion that, under conditions of severe malnutrition, photoperiodic time measurement continues in the growth-retarded female sheep. This suggests that the absence of puberty associated with gross undernutrition in the lamb most probably stems from inappropriate, internal growth-related cues rather than the inability to monitor 
external seasonal changes in daylength. Whether or not puberty will occur upon restitution of growth during increased nutrition depends upon the photoperiod history experienced by the young female.

We thank Mr Lee $\mathrm{H}$. Breasbois for providing high quality lambs for experimentation; $\mathrm{Mr}$ Douglas D. Doop for conscientious animal care and technical assistance; Ms Janice A. Clayton for hormone radioimmunoassays; Ms Diane E. Belleba for assistance in manuscript preparation; and Dr Gordon D. Niswender and Dr Leo E. Reichert, Jr for providing reagents used in hormone assays. This work was supported, in part, by NIH Grants HD-06471, HD-07048, HD-11311, HD-18258, and HD-18394 and Biomedical Research Support to the Vice President for Research of The University of Michigan.

\section{References}

Foster, D.L. (1984) Preovulatory gonadotropin surge system of prepubertal female sheep is exquisitely sensitive to the stimulatory feedback action of estradiol. Endocrinology 115, 1186-1189.

Foster, D.L. \& Olster, D.H. (1985) Effect of restricted nutrition on puberty in the lamb: patterns of tonic luteinizing hormone ( $\mathrm{LH})$ secretion and competency of the LH surge system. Endocrinology 116, 375-381.

Foster, D.L. \& Ryan, K.D. (1979) Endocrine mechanisms governing transition into adulthood: a marked decrease in inhibitory feedback action of estradiol on tonic secretion of luteinizing hormone in the lamb during puberty. Endocrinology 105, 896-904.

Foster, D.L., Yellon, S.M. \& Olster, D.H. (1985) Internal and external determinants of the timing of puberty in the female. J. Reprod. Fert. 75, 327-344.

Hauger, R.L., Karsch, F.J. \& Foster, D.L. (1977) A new concept for control of the estrous cycle of the ewe based on temporal relationships between luteinizing hormone, estradiol and progesterone in peripheral serum and evidence that progesterone inhibits tonic LH secretion. Endocrinology 101, 807-817.
Nicholls, T.J. \& Storey, C.R. (1976) The effects of castration on plasma LH levels in photosensitive and photorefractory canaries (Serinus canarius). Gen. comp. Endocr. 29, 170-174.

Niswender, G.D., Reichert, L.E., Jr, Midgley, A.R., Jr \& Nalbandov, A.V. (1969) Radioimmunoassay for bovine and ovine luteinizing hormone. Endocrinology 84, 1166-1173.

Yellon, S.M. \& Foster, D.L. (1985) Alternate photoperiods time puberty in the female lamb. Endocrinology 116, 2090-2097. 